\title{
UNSOLVABILITY CORES IN CLASSIFICATION PROBLEMS
}

\author{
ULRIKE BRANDT AND HERMANN K.-G. WALTER
}

Fachbereich Informatik, Technische Universität Darmstadt, Germany

e-mail address: brandt@dekanat.informatik.tu-darmstadt.de, walter@informatik.tu-darmstadt.de

\begin{abstract}
Classification problems have been introduced by M. Ziegler as a generalization of promise problems. In this paper we are concerned with solvability and unsolvability questions with respect to a given set or language family, especially with cores of unsolvability. We generalize the results about unsolvability cores in promise problems to classification problems. Our main results are a characterization of unsolvability cores via cohesiveness and existence theorems for such cores in unsolvable classification problems. In contrast to promise problems we have to strengthen the conditions to assert the existence of such cores. In general unsolvable classification problems with more than two components exist, which possess no cores, even if the set family under consideration satisfies the assumptions which are necessary to prove the existence of cores in unsolvable promise problems. But, if one of the components is fixed we can use the results on unsolvability cores in promise problems, to assert the existence of such cores in general. In this case we speak of conditional classification problems and conditional cores. The existence of conditional cores can be related to complexity cores. Using this connection we can prove for language families, that conditional cores with recursive components exist, provided that this family admits an uniform solution for the word problem.
\end{abstract}

\section{INTRODUCTION}

The concept of classsification problems was introduced by M. Ziegler ([1]) as a generalization of promise problems due to S. Even ([5]). Promise problems are a generalization of decision problems. A classification problem is a vector $\mathbf{A}=\left(A_{1}, \ldots, A_{k}\right)$ where the $A_{i}$ are pairwise disjoint infinite subsets of a given basic set $S$. For a set family $\mathcal{F} \subseteq \mathbf{2}^{S}$ such a classification problem is $\mathcal{F}$-solvable, if a vector $\mathbf{Q}=\left(Q_{1}, \ldots, Q_{k}\right)$ exists with $A_{i} \subseteq Q_{i}, Q_{i} \in \mathcal{F}, Q_{i} \cap Q_{j}=\emptyset$ for $1 \leq i \neq j \leq k$ and $Q_{1} \cup \cdots \cup Q_{k}=S$. If $k=2$ we are faced with promise problems. In applications $S=X^{*}$ where $X$ is a finite nonempty alphabet and $\mathcal{F}=\mathcal{L}$ a language family and/or a complexity class. From an algorithmic point of view solutions of classification problems can be used to obtain constant size advices. In this case advices indicate the inputs to belong to certain subsets (c.f. [1] for further details). We extend the results about unsolvability cores in promise problems ([4]) to unsolvability cores in classification problems. Again cohesiveness is the characterizing indicator. For unsolvable promise problems we can

$2012 A C M$ CCS: [Theory of computation]: Computational complexity and cryptographyComplexity classes.

Key words and phrases: Classification problems, conditional classification problems, cores of unsolvability, cohesiveness of sets and languages, recursive languages, complexity classes, hard cores.

DOI:10.2168/LMCS-10(2:12)2014

(c) U. Brandt and H. K.-G. Walter

(a) Creative Commons 
find in general unsolvability cores, if the set family is closed under union, intersection and finite variation. But for unsolvable classification problems with $k>2$ the existence of unsolvability cores needs further conditions. We show, that we can assert the existence of unsolvability cores for $k>2$ under the same assumption as needed for promise problems, if we fix one of the components. In this approach the fixed component is called the condition for the classification problem. The results are proven under assumptions which involve closure properties of $\mathcal{F}$ against some or all boolean operations union, intersection and complementation. Moreover, we can relate unsolvability cores for conditional classification problems to so called proper hard cores introduced by R. Book and D.-Z. Du in a general form ([3]) and first defined by N. Lynch ([6]) for complexity classes. Using results and proof techniques from [3] we can apply our results to language families and complexity classes. Especially, we are able to construct unsolvability cores where the components are recursive. To do this, the language family or complexity class under consideration must allow an enumeration where the word problem has a uniform solution. We assume the reader to be familiar with the theory of recursive functions, languages and complexity (cf.[2],[7]).

\section{Set and Language Families, Basic Notations}

In the following an infinite basic set $S$ is given. We assume that the elements of set families $\mathcal{F}$ are subsets of $\mathrm{S}$. Moreover, sets $A, A^{\prime}, B, B^{\prime}, C, \cdots, Q, \cdots$ are always subsets of $S$ and singletons $\{s\}$ are identified with $s$. We mainly deal with denumerable set families $\mathcal{F}$; i.e. a function $\mathbf{e}_{\mathcal{F}}: \mathbb{N}_{0} \rightarrow \mathbf{2}^{S}$ with $\mathbf{e}_{\mathcal{F}}\left(\mathbb{N}_{0}\right)=\mathcal{F}$ exists (enumeration of $\mathcal{F}$ ). Consider the boolean operations $A \cup B$ union, $A \cap B$ intersection and $A^{\mathbf{c}}=S \backslash A$ complementation in connection with set families $\mathcal{F}$. These operations can be lifted to binary operations between set families $\mathcal{F}_{1}$ and $\mathcal{F}_{2}$ and unary operations for $\mathcal{F}$. Define

$$
\begin{aligned}
& \mathcal{F}_{1} \oplus \mathcal{F}_{2}=\left\{A \cup B \mid A \in \mathcal{F}_{1} \text { and } B \in \mathcal{F}_{2}\right\}, \\
& \mathcal{F}_{1} \odot \mathcal{F}_{2}=\left\{A \cap B \mid A \in \mathcal{F}_{1} \text { and } B \in \mathcal{F}_{2}\right\}
\end{aligned}
$$

and the closure operations

$$
\begin{aligned}
& \mathcal{F}^{\mathbf{u}}=\left\{A_{1} \cup \ldots \cup A_{n} \mid n \geq 1, A_{i} \in \mathcal{F} \text { for } 1 \leq i \leq n\right\}(\text { union }), \\
& \mathcal{F}^{\mathbf{s}}=\left\{A_{1} \cap \ldots \cap A_{n} \mid n \geq 1, A_{i} \in \mathcal{F} \text { for } 1 \leq i \leq n\right\}(\text { inter section }), \\
& \mathcal{F}^{\mathbf{c o}}=\left\{A^{\mathbf{c}} \mid A \in \mathcal{F}\right\}, \mathcal{F}^{\mathbf{c c}}=\mathcal{F} \cup \mathcal{F}^{\mathbf{c o}}(\text { complementation }) \text { and } \\
& \left.\mathcal{F}^{\mathbf{b}}=\left(\left(\mathcal{F}^{\mathbf{c c}}\right)^{\mathbf{s}}\right)^{\mathbf{u}} \text { (boolean closure }\right) .
\end{aligned}
$$

We will frequently use $\mathcal{F}^{\mathbf{d c}}=\mathcal{F} \cap \mathcal{F}^{\mathbf{c o}}$. Note, that $\left(\mathcal{F}^{\mathbf{u}}\right)^{\mathbf{s}}=\left(\mathcal{F}^{\mathbf{s}}\right)^{\mathbf{u}}$ (distributivity $),\left(\mathcal{F}^{\mathbf{c o}}\right)^{\mathbf{u}}=$ $\left(\mathcal{F}^{\mathbf{s}}\right)^{\mathbf{c o}}($ deMorgan $),\left(\mathcal{F}^{\mathbf{c c}}\right)^{\mathbf{d c}}=\mathcal{F}^{\mathbf{c c}}$ and $\left(\mathcal{F}^{\mathbf{c o}}\right)^{\mathbf{c o}}=\mathcal{F}$. Furthermore, $\mathcal{F}=\mathcal{F}^{\mathbf{c c}}\left(\mathcal{F}=\mathcal{F}^{\mathbf{u}}\right.$, $\left.\mathcal{F}=\mathcal{F}^{\mathbf{s}}\right)$ if and only if $\mathcal{F}=\mathcal{F}^{\mathbf{c o}}(\mathcal{F} \oplus \mathcal{F} \subseteq \mathcal{F}, \mathcal{F} \odot \mathcal{F} \subseteq \mathcal{F}$, respectively $)$.

Let $\operatorname{fin}(S)=\{A \subseteq S \mid A$ finite $\}$. Then $\mathcal{F}$ is closed under finite variation if $\mathcal{F} \oplus \operatorname{fin}(S) \subseteq$ $\mathcal{F}$ and $\mathcal{F} \odot \boldsymbol{f i n}(S)^{\mathbf{c o}} \subseteq \mathcal{F}$. We call $\mathcal{F}$ nontrivial if $\emptyset, S \in \mathcal{F}$ and $\mathcal{F}$ is closed under finite variation. In this case $\operatorname{fin}(S) \subseteq \mathcal{F}$. Note, that $\operatorname{fin}(S)=\boldsymbol{f i n}(S)^{\mathbf{b}}$. Moreover, $\mathcal{F}^{\mathbf{c c}}, \mathcal{F}^{\mathbf{u}}, \mathcal{F}^{\mathbf{s}}$ and $\mathcal{F}^{\mathbf{b}}$ are nontrivial, if $\mathcal{F}$ is nontrivial.

Consider the case $S=X^{*}$, where $X^{*}$ is the free monoid over $X$ (a nonempty, finite alphabet) with concatenation of words as monoid operation and $\mathbf{1}$ as identity. As usual $L \subseteq X^{*}$ is called a language and $\mathcal{L} \subseteq \mathbf{2}^{X^{*}}$ a language family. For a word $w=x_{1} \ldots x_{n}\left(x_{i} \in X\right.$ for $1 \leq i \leq n)|w|=n$ is the length of $w$ and $|\mathbf{1}|=0$. For languages $L_{1}$ and $L_{2}$ the complex product is defined by $L_{1} L_{2}=\left\{w_{1} w_{2} \mid w_{1} \in L_{1}, w_{2} \in L_{2}\right\}$. There are various kinds 
of quotients available, for example the left quotient defined by $L_{1}^{-1} L_{2}=\left\{w \mid \exists w_{1} \in L_{1}\right.$ : $\left.w_{1} w \in L_{1}\right\}$. In this context we are mainly interested in handling leftmarkers, i.e. we consider the products $w L$ and the quotients $w^{-1} L$ where $w \in X^{*}$ and $L$ is a language. With respect to language families $\mathcal{L}$ we get the closure operations $\mathcal{L}^{\text {ltr }}=\left\{w L \mid w \in X^{*}, L \in \mathcal{L}\right\}$ and $\mathcal{L}^{\text {-ltr }}=\left\{w^{-1} L \mid w \in X^{*}, L \in \mathcal{L}\right\}$. In handling the leftmarkers (for example complementation of a leftmarked language) we use variation by $\mathcal{L}_{\text {reg }}(X)$, the family of regular languages (for details see [4]). A language family $\mathcal{L}$ is closed under regular variation if $\mathcal{L} \oplus \mathcal{L}_{\text {reg }}(X) \subseteq \mathcal{L}$ and $\mathcal{L} \odot \mathcal{L}_{\text {reg }}(X) \subseteq \mathcal{L}$.

Looking at (partial) orderings on $X^{*}$ the lexicographic ordering is important for our purposes. For $n \geq 0$ let $[n]_{0}=\{0, \ldots, n-1\}$ and $[n]=\{1, \ldots, n\}$. Given a bijection $\omega: X \rightarrow[b]_{0}(b=\#(X))$ define $w \leq v$ if and only if $(|w|<|v|$ or $(|w|=|v|$ and $\left(\forall u \in X^{*}, x, y \in X: w \in u x X^{*}\right.$ and $\left.\left.v \in u y X^{*} \Rightarrow \omega(x) \leq \omega(y)\right)\right)$. This is a well-ordering, hence we can define a successor function succ for $w \in X^{*}$ by $\operatorname{succ}(w)=\min \left\{v \in X^{*} \mid w \neq v\right.$ and $w \leq v\}$ where the minimum is taken with respect to the lexicographic ordering. Then $\lambda i . \boldsymbol{l e x}(i)=\boldsymbol{s u c c}^{i}(\mathbf{1})$ defines a bijection lex $: \mathbb{N}_{0} \rightarrow X^{*}$ with inverse ord $=$ lex $\boldsymbol{x}^{-1}$.

Consider the language families $\mathcal{L}_{\text {r.e. }}(X)$ (recursively enumerable languages) and $\mathcal{L}_{\text {rec }}(X)$ $=\mathcal{L}_{\text {r.e. }}(X)^{\mathbf{d c}}$ (recursive languages). Let $\boldsymbol{r e c}_{n}(n \geq 0)$ be the set of $n$-ary recursive functions. Using $0,1 \in \mathbb{N}_{0}$ as truth values define for a language $L$ the function $\lambda i . \delta_{L}(i)=$ "lex $(i) \in L$ ". Then a language $L$ is recursive if and only if $\delta_{L} \in \boldsymbol{r e c}_{1}$. Alternatively, a nonempty language $L$ is recursive if and only if a function $f: \mathbb{N}_{0} \rightarrow X^{*}$ exists such that $\lambda i$.ord $(f(i))$ is nondecreasing and recursive. Classical language families and complexity classes are always denumerable. Of special interest are families with enumerations which are in a certain sense "effective". For our purpose it is important to assert that these enumerations allow a uniform solution for the word problem. More formular, we define for an enumeration $\mathbf{e}$ of a language family $\mathcal{L}$ the function $\lambda i, j$. $\boldsymbol{w o r d}_{\mathbf{e}}(i, j)=" \boldsymbol{l e x}(j) \in \mathbf{e}(i)$ ". If $\boldsymbol{w o r d}_{\mathbf{e}} \in \boldsymbol{r e c}_{2}$ then $\mathbf{e}$ is called WP-recursive. $\mathcal{L}$ is called WP-recursive, if a WP-recursive enumeration $\mathbf{e}$ of $\mathcal{L}$ exists. Note, that any WP-recursive $\mathcal{L}$ is a (proper) subfamily of $\mathcal{L}_{\text {rec }}(X)$ and every complexity class with reasonable ressource bounds (time- and space-constructability [2]) is WP-recursive.

\section{Solvability of Classification Problems}

Let $k>0$. We consider vectors $\mathbf{A}=\left(A_{1}, \ldots, A_{k}\right)$ with $A_{i} \subseteq S$ for $1 \leq i \leq k$. To such an $\mathbf{A}$ we associate two functions $\operatorname{set}(\mathbf{A})=A_{1} \cup \cdots \cup A_{k}$ and $|\mathbf{A}|=k$. Moreover, if $\mathbf{B}=\left(B_{1}, \ldots, B_{m}\right)$ with $1 \leq m \leq k$ is another vector, then $\mathbf{B} \leq \mathbf{A}$ if and only if an injective $\sigma:[m] \rightarrow[k]$ exists with $B_{i} \subseteq A_{\sigma(i)}$ for $1 \leq i \leq m$. A is a classification problem if $A_{i}$ is infinite and $A_{i} \cap A_{j}=\emptyset$ for all $1 \leq i \neq j \leq k$. For a given $\mathcal{F}$ a vector $\mathbf{Q}=\left(Q_{1}, \ldots, Q_{k}\right)$ is an $\mathcal{F}$-partition if $\operatorname{set}(\mathbf{Q})=S, Q_{i} \in \mathcal{F}$ and $Q_{i} \cap Q_{j}=\emptyset$ for $1 \leq i \neq j \leq k$.

Definition 2.1. A classification problem $\mathbf{A}$ is $\mathcal{F}$-solvable $\left(A \in \operatorname{class}_{k}(\mathcal{F})\right)$ if and only if an $\mathcal{F}$-partition $\mathbf{Q}$ exists with $|\mathbf{Q}|=k$ and $\mathbf{A} \leq \mathbf{Q}$, where $k=|\mathbf{A}|$.

If $S=\mathbb{N}_{0}$ then $\mathcal{F}$-solvability of promise problems corresponds to the separation principle defined in [7] (exercise 5-33). Our definition of $\mathcal{F}$-solvability for classification problems is stronger than the definition of $\mathcal{F}$-separability given in [1], where a classification problem $\mathbf{A}$ is $\mathcal{F}$-separable, if there exists a $\mathbf{Q}$, which satisfies the conditions of Definition 2.1. except the condition "set $(\mathbf{Q})=S$ ", which may not necessarily be valid. Note that for such a $\mathbf{Q}$, we always obtain $Q_{k} \subseteq\left(Q_{1} \cup \cdots \cup Q_{k-1}\right)^{\mathbf{c}}$. Hence, the class of $\mathcal{F}$-solvable classification problems 
with more than one components is identical with the class of $\mathcal{F}$-separable classification problems, if $\mathcal{F}$ is a boolean algebra. That $\mathcal{F}$-solvability is stronger than $\mathcal{F}$-separability, follows from results in [7]. Consider $\mathcal{L}_{\text {r.e. }}(X)$ where $\mathrm{X}$ is a one-letter alphabet. Then a promise problem $(A, B)$ consisting of recursively enumerable sets exists, which is not $\mathcal{L}_{\text {r.e. }}(X)$-solvable $([7]$ exercise $5-34)$. But $(A, B)$ is clearly $\mathcal{L}_{\text {r.e. }}(X)$-separable. We also find the interesting result that any promise problem $(A, B)$ with $A, B \in \mathcal{L}_{\text {r.e. }}(X)^{\text {co }}$ is $\mathcal{L}_{\text {r.e. }}(X)^{\text {co }}$ solvable ([7] exercise 5-33). Hence all promise problems, which are $\mathcal{L}_{\text {r.e. }}(X)^{\text {co }_{\text {-separable }} \text { are }}$ $\mathcal{L}_{\text {r.e. }}(X)^{\mathbf{c o}}$-solvable. But $\mathcal{L}_{\text {r.e. }}(X)^{\text {co }}$ is not closed under complementation.

For $k=1$ we identify $A_{1}$ with $\left(A_{1}\right)$. If $\mathcal{F}$ is nontrivial then every $A_{1}$ is $\mathcal{F}$-solvable. If $k>2$ and $\mathcal{F}$ satisfies appropriate closure properties, then we can reduce the question of solvability of classification problems to solvability of promise problems. Directly from the definition we get

Proposition 2.2. If $\mathcal{F}=\mathcal{F}^{u}$ then for all classification problems $\boldsymbol{A}$ and $\boldsymbol{B}$ with $\boldsymbol{B} \leq \boldsymbol{A}$ $\boldsymbol{A} \in \operatorname{class}_{|\boldsymbol{A}|}(\mathcal{F})$ implies $\boldsymbol{B} \in \operatorname{class}_{|\boldsymbol{B}|}(\mathcal{F})$.

Proof. Suppose $\mathbf{B} \leq \mathbf{A} \leq \mathbf{Q}$ where $\mathbf{Q}$ is an $\mathcal{F}$-partition. Let $B=\left(B_{1}, \ldots, B_{m}\right), A=$ $\left(A_{1}, \ldots, A_{k}\right)$ and $Q=\left(Q_{1}, \ldots, Q_{k}\right)$. Then we can assume without loss of generality $B_{i} \subseteq$ $A_{i} \subseteq Q_{i}$ for all i. Consider $\mathrm{P}=Q_{1} \cup \cdots \cup Q_{k}$. Then $P^{\mathbf{c}}=Q_{m+1} \cup \cdots \cup Q_{k} \in \mathcal{F}$. Hence, $\mathbf{Q}^{\prime}=\left(Q_{1}, \ldots, Q_{k-1}, Q_{k} \cup P^{\mathbf{c}}\right)$ is an $\mathcal{F}$-partition with $\mathbf{B} \leq \mathbf{Q}^{\prime}$.

Lemma 2.3. If $\mathcal{F}=\mathcal{F}^{u}=\mathcal{F}^{s}$ and $\boldsymbol{A}=\left(A_{1}, \ldots A_{k}\right)$ is a classification problem then $\boldsymbol{A} \in \operatorname{class}_{k}(\mathcal{F})$ if and only if $\left(A_{i}, A_{j}\right) \in \operatorname{class}_{2}(\mathcal{F})$ for all $1 \leq i \neq j \leq k$.

Proof. The "if part" follows by Proposition 2.2. Suppose that $\left(A_{i}, A_{j}\right) \in \operatorname{class}_{2}(\mathcal{F})$ for $1 \leq i \neq j \leq k$. Now we proceed by induction over $|\mathbf{A}|=k$. If $\mathrm{k}=2$ nothing is to prove. Let $\mathbf{A}=\left(A_{1}, \ldots, A_{k+1}\right)$ and suppose $\left(A_{1}, \ldots, A_{k}\right) \in \operatorname{class}_{k}(\mathcal{F})$. Then an $\mathcal{F}$-partition $\mathbf{Q}^{\prime}=\left(Q_{1}^{\prime}, \ldots, Q_{k}^{\prime}\right)$ with $\left(A_{1}, \ldots, A_{k}\right) \leq \mathbf{Q}^{\prime}$ exists. Assume without loss of generality $A_{i} \subseteq Q_{i}^{\prime}$ for $1 \leq i \leq k$. On the other side $Q_{i}^{\prime \prime} \in \mathcal{F}^{\text {dc }}$ exist with $A_{i} \subseteq Q_{i}^{\prime \prime}$ and $A_{k+1} \subseteq\left(Q_{i}^{\prime \prime}\right)^{\mathbf{c}}$ for $1 \leq i \leq k$. Consider $P=Q_{1}^{\prime \prime} \cup \cdots \cup Q_{k}^{\prime \prime}$. Then $A_{i} \subseteq P \in \mathcal{F}$ for $1 \leq i \leq k$ and $P^{\mathbf{c}}=\left(Q_{1}^{\prime \prime}\right)^{\mathbf{c}} \cap \cdots \cap\left(Q_{k}^{\prime \prime}\right)^{\mathbf{c}} \in \mathcal{F}$ with $A_{k+1} \subseteq P^{\mathbf{c}}$. This shows $\mathbf{Q}=\left(Q_{1}^{\prime} \cap P, \ldots, Q_{k}^{\prime} \cap P, P^{\mathbf{c}}\right)$ is an $\mathcal{F}$-partition with $\mathbf{A} \leq \mathbf{Q}$.

As indicated in the introduction we generalize the notion of a classification problem to conditional classification problems by fixing one component as condition. Consider $C \subseteq$ $S$ and a classification problem $\mathbf{A}$. Then $(C, \mathbf{A})$ is a conditional classification problem if $C \cap \operatorname{set}(\mathbf{A})=\emptyset$, referring to $C$ as the problem condition. $C$ could be finite, even empty. If $C^{\mathbf{c}}$ is finite, then no conditional classification problems $(C, \mathbf{A})$ exist.

Definition 2.4. A conditional classification problem $(C, \mathbf{A})$ is called $\mathcal{F}$-solvable $(A \in$ $\left.\operatorname{cclass}_{k}(C, \mathcal{F})\right)$ if and only if an $\mathcal{F}$-partition $\mathbf{Q}=\left(Q_{0}, Q_{1}, \ldots, Q_{k}\right)$ exists with $C \subseteq Q_{0}$ and $\mathbf{A} \leq\left(Q_{1}, \ldots, Q_{k}\right)$ where $k=|\mathbf{A}|$.

The following facts follow directly from the definition

Proposition 2.5. Let $\mathcal{F}$ and $k>0$ be given.

(1) $C_{1} \subseteq C_{2} \subseteq S \Rightarrow \operatorname{cclass}_{k}\left(C_{2}, \mathcal{F}\right) \subseteq \operatorname{cclass}_{k}\left(C_{1}, \mathcal{F}\right)$.

(2) $C^{c} \in \operatorname{fin}(S) \Rightarrow \operatorname{cclass}_{k}(C, \mathcal{F})=\emptyset$.

(3) $\emptyset \in \mathcal{F} \Rightarrow \operatorname{class}_{k}(\mathcal{F}) \subseteq \operatorname{cclass}_{k}(\emptyset, \mathcal{F})$.

(4) $\mathcal{F}=\mathcal{F}^{u} \Rightarrow \operatorname{class}_{k}(\mathcal{F})=\operatorname{cclass}_{k}(\emptyset, \mathcal{F})$.

(5) $\mathcal{F}$ nontrivial and $C \in \operatorname{fin}(S) \Rightarrow \operatorname{cclass}_{k}(C, \mathcal{F})=\operatorname{cclass}_{k}(\emptyset, \mathcal{F})$. 
Example 2.6. Consider $X=\{a, b\}$. Let $\mathcal{L}=\mathcal{L}^{\text {ltr }}=\mathcal{L}^{\text {-ltr }}$ a nontrivial language family, which is closed under regular variation. If $A$ is a set with $A^{\mathbf{c}}, A \notin \mathcal{L}$, then $\left(A^{\mathbf{c}}, A\right) \notin$ $\operatorname{class}_{2}(\mathcal{L})$ and by our assumption on $\mathcal{L}\left(x A^{\mathbf{c}}, x A\right) \notin \operatorname{class}_{2}(\mathcal{L})$ for $x=a, b$ (Lemma 5.4. in [4]). Clearly, $\left(a A^{\mathbf{c}}, b A\right) \in \operatorname{class}_{2}(\mathcal{L})$, but $\left(a A \cup b A^{\mathbf{c}}, a A^{\mathbf{c}}, b A\right) \notin \operatorname{class}_{3}(\mathcal{L})$. Hence $\left(a A^{\mathbf{c}}, b A\right) \notin \operatorname{cclass}_{2}\left(a A \cup b A^{\mathbf{c}}, \mathcal{L}\right)$.

\section{Unsolvability Cores in Classification Problems}

As in the case of promise problems unsolvability of classification problems is closely related to cohesiveness.

Definition 3.1. $A \subseteq S$ is $\mathcal{F}$-cohesive $(A \in$ cohesive $(\mathcal{F}))$ if and only if $A$ is infinite and for all $Q \in \mathcal{F}^{\text {dc }}$ either $A \cap Q$ or $A \cap Q^{\mathbf{c}}$ is finite (cf.[4] and [7]).

Remark 3.2. It is interesting to compare our definition of cohesiveness with related classical definitions, as they are presented in [7]. Consider the families $\mathcal{L}_{\text {r.e. }}(X)^{\text {cc }}, \mathcal{L}_{\text {r.e. }}(X)$ and $\mathcal{L}_{\text {rec }}(X)$. Then $L \in$ cohesive $\left(\mathcal{L}_{\text {r.e. }}(X)^{\text {cc }}\right)$ if and only if $L$ is cohesive in the classical sense. Moreover, cohesive $\left(\mathcal{L}_{\text {rec }}(X)\right)=\operatorname{cohesive}\left(\mathcal{L}_{\text {r.e. }}(X)\right)$, since a language $Q$ is recursive if and only if $Q$ and $Q^{\mathbf{c}}$ are recursively enumerable. Furthermore the definition of recursively indecomposability coincides with the definition of $\mathcal{L}_{\text {rec }}(X)$-cohesiveness. In [7] we also find the notion of indecomposability. $L$ is indecomposable if there exist no infinite sets $L_{1}, L_{2} \in \mathcal{L}_{\text {r.e. }}(X)$ such that $L_{1} \cap L_{2}=\emptyset, L \subseteq L_{1} \cup L_{2}, L \cap L_{1}$ is infinite and $L \cap L_{2}$ is infinite. Then we find the following results in [7]. If $L \in \operatorname{cohesive}\left(\mathcal{L}_{\text {r.e. }}(X)^{\mathbf{c c}}\right)$ then it is indecomposable and any indecomposable $L$ is $\mathcal{L}_{\text {rec }}(X)$-cohesive. None of the converse implications hold.

In [4] (Theorem 5.1.) it is proven, that for a promise problem $(A, B)$ and a nontrivial set family $\mathcal{F} A \cup B \in \operatorname{cohesive}(\mathcal{F})$ if and only if $A, B \in \operatorname{cohesive}(\mathcal{F})$ and $(A, B) \notin \operatorname{class}_{2}(\mathcal{F})$. This result leads to a much stronger one. In the theory of complexity we find the notion of hard cores inside those sets which can be computed with bounded ressources (time, space, e.t.c. [3]). Similarily, we can consider unsolvability cores of classification problems which are not solvable.

Definition 3.3. For $k>1$ a classification problem $\mathbf{A}$ with $|\mathbf{A}|=k$ is a $k$-core of $\mathcal{F}$ $\left(\mathbf{A} \in \operatorname{core}_{k}(\mathcal{F})\right)$ if and only if for all classification problems $\mathbf{A}^{\prime}$ with $\mathbf{A}^{\prime} \leq \mathbf{A}$ and $\left|\mathbf{A}^{\prime}\right|>1$ : $\mathbf{A}^{\prime} \notin \operatorname{class}_{\left|\mathbf{A}^{\prime}\right|}(\mathcal{F})$.

Clearly, any subproblem of a core is itself a core. This is especially true for subproblems, which are promise problems. This enables us to use the results about unsolvability cores for promise problems from [4].

Lemma 3.4. If $\mathcal{F}=\mathcal{F}^{u}$ and $\boldsymbol{A}=\left(A_{1}, \ldots, A_{k}\right)(k>1)$ is a classification problem then $\boldsymbol{A} \in \operatorname{core}_{k}(\mathcal{F})$ if and only if $\left(A_{i}, A_{j}\right) \in$ core $_{2}(\mathcal{F})$ for all $1 \leq i \neq j \leq k$.

Proof. Suppose $\mathbf{A} \in \operatorname{core}_{k}(\mathcal{F})$, then by definition $\left(A_{i}, A_{j}\right) \leq \mathbf{A}$ and therefore $\left(A_{i}, A_{j}\right) \in$ core $_{2}(\mathcal{F})$. Conversely, suppose that $\mathbf{A} \notin \operatorname{core}_{k}(\mathcal{F})$, i. e. $\mathbf{A}^{\prime}=\left(A_{1}^{\prime}, \ldots, A_{m}^{\prime}\right)$ exists with $\mathbf{A}^{\prime} \leq \mathbf{A}, m>1$ and $\mathbf{A}^{\prime} \in \operatorname{class}_{\left|\mathbf{A}^{\prime}\right|}(\mathcal{F})$. Since $\mathcal{F}=\mathcal{F}^{\mathbf{u}}$ we know $\left(A_{1}^{\prime}, A_{2}^{\prime}\right) \in \operatorname{class}_{2}(\mathcal{F})$. Moreover, $A_{1}^{\prime} \subseteq A_{i}$ and $A_{2}^{\prime} \subseteq A_{j}$ for some $1 \leq i \neq j \leq k$. But then $\left(A_{i}, A_{j}\right) \notin \operatorname{core}_{2}(\mathcal{F})$. 
Now we can characterize cores by cohesiveness. Using Theorem 5.1. and Theorem 6.7. of [4] we can prove

Theorem 3.5. If $\mathcal{F}=\mathcal{F}^{u}$ is nontrivial and $\boldsymbol{A}$ a classification problem with $|\boldsymbol{A}|=k>1$ then $\boldsymbol{A} \in \operatorname{core}_{k}(\mathcal{F})$ if and only if $\operatorname{set}(\boldsymbol{A}) \in \operatorname{cohesive}(\mathcal{F})$.

Proof. If $\mathbf{A}=\left(A_{1}, \ldots, A_{k}\right) \in$ core $_{k}(\mathcal{F})$, then $\left(A_{i}, A_{j}\right) \in$ core $_{2}(\mathcal{F})$ for all $1 \leq i \neq j \leq k$. By Theorem 6.7. in [4] we know $A_{1} \cup A_{i} \in \operatorname{cohesive}(\mathcal{F})$ for all $2 \leq i \leq k$. But then $A_{1} \cup \cdots \cup A_{k}=\left(A_{1} \cup A_{2}\right) \cup \cdots \cup\left(A_{1} \cup A_{k}\right)$. Since $A_{1} \subseteq\left(A_{1} \cup A_{i}\right) \cap\left(A_{1} \cup A_{j}\right)$ for all $2 \leq i \neq j \leq k$ and $A_{1}$ is infinite, a simple induction proof shows $\operatorname{set}(\mathbf{A}) \in \operatorname{cohesive}(\mathcal{F})$.

Conversely, if $A_{1} \cup \cdots \cup A_{k} \in \operatorname{cohesive}(\mathcal{F})$ then for all $1 \leq i \neq j \leq k, A_{i} \cup A_{j} \in$ cohesive $(\mathcal{F})$. Again by Theorem 6.7. of $[4]\left(A_{i}, A_{j}\right) \in$ core $_{2}(\mathcal{F})$ and therefore by Lemma 3.4. $\mathbf{A} \in \operatorname{core}_{k}(\mathcal{F})$.

We can find to any classification problem $\mathbf{A}$ with $|\mathbf{A}|=2$ and $\mathbf{A} \notin \operatorname{class}_{2}(\mathcal{F})$ a $\mathbf{B} \leq \mathbf{A}$ such that $\mathbf{B} \in \operatorname{core}_{2}(\mathcal{F})$ if $\mathcal{F}=\mathcal{F}^{\mathbf{u}}=\mathcal{F}^{\mathbf{s}}$ is denumerable ([4]). But this is not true for classification problems $\mathbf{A}$ with $|\mathbf{A}|>2$. To see this we prove the following theorem, where we use $S=X^{*}$ with $X=\{a, b, c\}$. Define for $A \subseteq X^{*}$ the classification problem $\mathbf{C}(A)=\left(A_{a b}, A_{b c}, A_{c a}\right)$, where $A_{x y}=x A \cup y A^{\mathbf{c}}$ for $x, y \in X$.

Theorem 3.6. Let $\mathcal{L}$ be a nontrivial language family with $\mathcal{L}=\mathcal{L}^{u}=\mathcal{L}^{\text {ltr }}=\mathcal{L}^{\text {-ltr }}$, which is closed under regular variation. If $A \subseteq S$ with $A \notin \mathcal{L}$ or $A^{c} \notin \mathcal{L}$, then $C(A) \notin \operatorname{class}_{3}(\mathcal{L})$ and for all $\boldsymbol{B} \leq \boldsymbol{C}(A)$ with $|\boldsymbol{B}|=3: \boldsymbol{B} \notin$ core $_{3}(\mathcal{L})$.

Proof. (1) We know $\left(A^{\mathbf{c}}, A\right) \notin$ class $_{2}(\mathcal{L})([4])$. But then by Lemma 5.4. of $[4]\left(x A^{\mathbf{c}}, x A\right) \notin$ $\operatorname{class}_{2}(\mathcal{L})$ for all $x \in X$. Now $\left(b A^{\mathbf{c}}, b A\right) \leq\left(A_{a b}, A_{b c}\right),\left(c A^{\mathbf{c}}, c A\right) \leq\left(A_{b c}, A_{c a}\right)$ and $\left(a A^{\mathbf{c}}, a A\right) \leq\left(A_{c a}, A_{a b}\right)$. This shows $\left(A_{x y}, A_{x z}\right) \notin \operatorname{class}_{2}(\mathcal{L})$ for all $x \neq y, z \neq y$ and $x \neq z$.

(2) Suppose $\mathbf{B} \leq \mathbf{C}(A)$ exists with $\mathbf{B} \in \operatorname{core}_{3}(\mathcal{L})$. Then by Theorem 3.5. $\boldsymbol{s e t}(\mathbf{B}) \in$ cohesive $(\mathcal{L})$. Assume without loss of generality that $\mathbf{B}=(B(a, b), B(b, c), B(c, a))$ and $B(x, y) \subseteq A_{x y}$ for $x, y \in X$ with $x \neq y$.. In the following let $B^{\prime}(x, y)=B(x, y) \cap x X^{*}$ and $B^{\prime \prime}(x, y)=B(x, y) \cap\left(x X^{*}\right)^{\mathbf{c}}$.

Assertion : $B^{\prime}(x, y) \in \boldsymbol{f i n}\left(X^{*}\right)$ for all $x, y \in X$ with $x \neq y$. Suppose to the contrary (without loss of generality) $B^{\prime}(a, b) \notin \operatorname{fin}\left(X^{*}\right)$. But then $B^{\prime}(b, c) \in$ $\boldsymbol{f i n}\left(X^{*}\right)$. Otherwise we obtain $\left(B^{\prime}(a, b), B^{\prime}(b, c)\right) \leq\left(a X^{*}, b X^{*}\right) \leq\left(a X^{*},\left(a X^{*}\right)^{\mathbf{c}}\right)$. Since $\mathcal{L}_{\text {reg }} \subseteq \mathcal{L}, \mathbf{B} \notin \operatorname{core}_{3}(\mathcal{L})$ - a contradiction. But now $B^{\prime \prime}(b, c)$ is infinite and $B^{\prime \prime}(b, c) \subseteq$ $c X^{*} \subseteq\left(a X^{*}\right)^{\mathbf{c}}$, hence both $\operatorname{set}(\mathbf{B}) \cap a X^{*}$ and $\operatorname{set}(\mathbf{B}) \cap\left(a X^{*}\right)^{\mathbf{c}}$ are infinite - a contradiction to $\operatorname{set}(\mathbf{B}) \in \operatorname{cohesive}(\mathcal{L})$.

Now consider $B^{\prime \prime}(a, b)$ and $B^{\prime \prime}(c, a)$. Then both sets are infinite and $\left(B^{\prime \prime}(a, b), B^{\prime \prime}(c, a)\right) \leq$ $\left(b X^{*}, a X^{*}\right) \leq\left(b X^{*},\left(b X^{*}\right)^{\mathbf{c}}\right)$ - a contradiction to $\mathbf{B} \in \operatorname{core}_{3}(\mathcal{L})$. This completes the proof.

Remark 3.7. The basic idea behind the proof of Theorem 3.6. is due to M. Ziegler ([1]). Note, that complexity classes and most of the known language families satisfy the conditions of Theorem 3.6.

Using conditional unsolvability, we can derive an existence theorem for cores.

Theorem 3.8. Let $\mathcal{F}=\mathcal{F}^{u}=\mathcal{F}^{s}$ be denumerable and nontrivial. If $\boldsymbol{A}=\left(A_{1}, \ldots, A_{k}\right)$ is a classification problem and $C \subseteq \operatorname{set}(\boldsymbol{A})^{c}$ is $\mathcal{F}$-cohesive with $\left(C, A_{i}\right) \notin \operatorname{class}_{2}(\mathcal{F})$ for $1 \leq i \leq k$, then there exists $\boldsymbol{B} \leq \boldsymbol{A}$ with $|\boldsymbol{B}|=k$ and $\boldsymbol{B} \in \operatorname{core}_{k}(\mathcal{F})$. 
Proof. Since $\left(C, A_{i}\right) \notin$ class $_{2}(\mathcal{F})$, we can find $C_{i} \subseteq C$ and $B_{i} \subseteq A_{i}$ with $\left(C_{i}, B_{i}\right) \in$ core $_{2}(\mathcal{F})$ (Theorem 6.14. in [4]). By Theorem 3.5. $C_{i} \cup B_{i} \in$ cohesive $(\mathcal{F})$ and therefore $B_{i} \in$

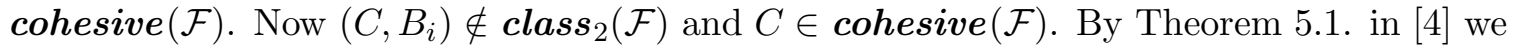
know $C \cup B_{i} \in$ cohesive $(\mathcal{F})$. But then $C \cup B_{1} \cup \cdots \cup B_{k}=\left(C \cup B_{1}\right) \cup \cdots \cup\left(C \cup B_{k}\right) \in$ cohesive $(\mathcal{F})$, since for all $1 \leq i \neq j \leq k C$ is infinite and $C \subseteq\left(C \cup B_{i}\right) \cap\left(C \cup B_{j}\right)$. It follows $B_{1} \cup \cdots \cup B_{k} \in$ cohesive $(\mathcal{F})$ and we obtain $\mathbf{B}=\left(B_{1}, \ldots, B_{k}\right) \leq \mathbf{A}$ and by Theorem 3.5. $\mathrm{B} \in \operatorname{core}_{k}(\mathcal{F})$.

Remark 3.9. Consider the situation of Theorem 3.6. Then $\operatorname{set}(\mathbf{C}(A))=X X^{*}$ and there is no room for an infinite condition $C$ to make the conditional classification problem $(C, \mathbf{C}(A))$ $\mathcal{L}$-solvable.

\section{Cores in Conditional Classification Problems}

Unsolvability of conditional classification problems can be related to cohesiveness, too.

Definition 4.1. Let $C, A \subseteq S$. Then $A$ is $\mathcal{F}$-cohesive under condition $C$ (in short: $A \in$ ccohesive $(C, \mathcal{F})$ ), if and only if $A$ is infinite and for all $Q \in \mathcal{F}^{\mathrm{dc}}$ with $Q \subseteq C$ either $A \cap Q$ or $A \cap Q^{c}$ is finite.

Clearly, if $C_{1} \subseteq C_{2} \subseteq S$, then ccohesive $\left(C_{2}, \mathcal{F}\right) \subseteq$ ccohesive $\left(C_{1}, \mathcal{F}\right)$. Especially, we get ccohesive $(S, \mathcal{F})=\operatorname{cohesive}(\mathcal{F})$ and therefore cohesive $(\mathcal{F}) \subseteq \operatorname{ccohesive}(C, \mathcal{F})$ for all $C \subseteq S$. Rewriting the definition, we also find $\operatorname{ccohesive}(C, \mathcal{F}))=\operatorname{cohesive}\left(\mathcal{F}(C)^{\mathbf{c c}}\right)$ where $\mathcal{F}(C)=\{Q \mid Q \subseteq C$ and $Q \in \mathcal{F}\}$. Analogously, we define conditional cores by

Definition 4.2. Let $C \subseteq S$ and $\mathbf{A}$ a classification problem. Then $\mathbf{A}$ is a $C$-conditional core of $\mathcal{F}\left(\mathbf{A} \in\right.$ ccore $\left._{|\mathbf{A}|}(C, \mathcal{F})\right)$ if and only if for all $\mathbf{A}^{\prime} \leq \mathbf{A}$ with $\left|\mathbf{A}^{\prime}\right|>0: \mathbf{A}^{\prime} \notin \operatorname{cclass}_{\left|\mathbf{A}^{\prime}\right|}(C, \mathcal{F})$.

In contrast to the definition of $\operatorname{core}(\mathcal{F})$ subproblems $\mathbf{A}^{\prime}$ with $\left|\mathbf{A}^{\prime}\right|=1$ are considered, too. Note, that $\left(C, \mathbf{A}^{\prime}\right)$ is a conditional-classification problem, if $\mathbf{A}^{\prime} \leq \mathbf{A}$. Moreover, if $\mathbf{A} \in$ ccore $_{|\mathbf{A}|}(C, \mathcal{F})$, then $\mathbf{A}^{\prime} \in$ ccore $_{\left|\mathbf{A}^{\prime}\right|}(C, \mathcal{F})$. The following lemma characterizes $A \in$ ccore $_{1}(C, \mathcal{F})$ by conditional cohesiveness.

Lemma 4.3. Let $\mathcal{F}$ be nontrivial and $C, A \subseteq S$ with $A$ infinite and $A \cap C=\emptyset$. Then the following statements are equivalent

(i) $A \in$ ccore $_{1}(C, \mathcal{F})$

(ii) $A \notin \operatorname{cclass}_{1}(C, \mathcal{F})$ and $A \in \operatorname{ccohesive}\left(C^{c}, \mathcal{F}\right)$.

Proof. (i) $\Rightarrow$ (ii): Suppose $A \in \operatorname{ccore}_{1}(C, \mathcal{F})$. Then $A \notin \operatorname{cclass}_{1}(C, \mathcal{F})$. Assume to the contrary that $A \notin$ ccohesive $\left(C^{\mathbf{c}}, \mathcal{F}\right)$. Then $Q \in \mathcal{F}^{\text {dc }}$ exists with $Q \subseteq C^{\mathbf{c}}, A \cap Q \notin \operatorname{fin}(S)$ and $A \cap Q^{\mathbf{c}} \notin \operatorname{fin}(S)$. Let $B=A \cap Q$. Then $B \subseteq Q$, but $Q \subseteq C^{\mathbf{c}}$, hence $C \subseteq Q^{\mathbf{c}}$. Moreover, $Q, Q^{\mathbf{c}} \in \mathcal{F}$, i.e. $B \in \operatorname{cclass}_{1}(C, \mathcal{F})$.

(ii) $\Rightarrow\left(\right.$ i): Suppose that $A \notin \operatorname{cclass}_{1}(C, \mathcal{F})$ and $A \in \operatorname{ccohesive}\left(C^{\mathbf{c}}, \mathcal{F}\right)$. Assume to the contrary that an infinite set $B \subseteq A$ exists, such that $B \subseteq Q^{\mathbf{c}}$ and $C \subseteq Q$ for some $Q \in \mathcal{F}^{\text {dc }}$. Then $Q^{\mathbf{c}} \subseteq C^{\mathbf{c}}$. Since $B \cap Q^{\mathbf{c}} \notin \operatorname{fin}(S), A \cap Q^{\mathbf{c}} \notin \operatorname{fin}(S)$, too. Hence $A \cap Q \in \operatorname{fin}(S)$, because $A \in$ ccohesive $\left(C^{\mathbf{c}}, \mathcal{F}\right)$. Consider $Q^{\prime}=Q^{\mathbf{c}} \cup(A \cap Q)$. Since $\mathcal{F}$ is nontrivial, $Q^{\prime} \in \mathcal{F}$. Note that $A=(A \cap Q) \cup\left(A \cap Q^{\mathbf{c}}\right) \subseteq Q^{\mathbf{c}} \cup(A \cap Q)=Q^{\prime}$. On the other side, $Q^{\mathbf{c}} \subseteq C^{c}$ and $A \cap Q \subseteq A \subseteq C^{\mathbf{c}}$, i.e. $Q^{\prime} \subseteq C^{\mathbf{c}}$. Hence $C \subseteq Q^{\prime \mathbf{c}}$. This shows that $A \notin \operatorname{cclass}_{1}(C, \mathcal{F})$ - a contradiction. 
Theorem 4.4. Let $\mathcal{F}$ be nontrivial with $\mathcal{F}=\mathcal{F}^{u}$ and $(C, \boldsymbol{A})$ a conditional $k$-classification problem. If $\boldsymbol{A}=\left(A_{1}, \ldots, A_{k}\right)$ then the following statements are equivalent

(i) $\boldsymbol{A} \in$ ccore $_{k}(C, \mathcal{F})$

(ii) $A_{i} \notin \operatorname{cclass}_{1}(C, \mathcal{F})$ and $A_{i} \in \operatorname{ccohesive}\left(C^{c}, \mathcal{F}\right)$ for all $1 \leq i \leq k$.

Proof.

(i) $\Rightarrow$ (ii): Suppose that $\mathbf{A} \in$ ccore $_{k}(C, \mathcal{F})$. Then for all $1 \leq i \leq k:\left(C, A_{i}\right) \in$ ccore $_{1}(C, \mathcal{F})$, since $A_{i} \leq \mathbf{A}$. Applying Lemma 4.3. we get the result.

(ii) $\Rightarrow$ (i): Let the $A_{i}$ be given according to the assumption. Assume to the contrary that $\mathbf{B} \leq \mathbf{A}$ exists with $\mathbf{B}=\left(B_{1}, \ldots, B_{m}\right) \in \operatorname{cclass}_{m}(C, \mathcal{F})$. Then an injective $\sigma:[m] \rightarrow[k]$ exists with $B_{i} \subseteq A_{\sigma(i)}$ for $1 \leq i \leq k$. Since $\mathcal{F}=\mathcal{F}^{\mathbf{u}}, B_{i} \in \operatorname{cclass}_{1}(C, \mathcal{F})$. But $A_{\sigma(i)} \in$ core $_{1}(C, \mathcal{F})$ and $B_{i} \subseteq A_{\sigma(i)}$. This is a contradiction.

Now, we are able to assert the existence of conditional cores in the case that both $C$ and $C^{\mathbf{c}}$ are infinite. Observe that under this assumption $A \in \operatorname{cclass}_{1}(C, \mathcal{F})$ if and only if $(C, A)$ considered as a promise problem is solvable for $\mathcal{F}$, i.e. $(C, A) \in \operatorname{class}_{1}(\mathcal{F})$.

Lemma 4.5. Let $\mathcal{F}$ be denumerable and nontrivial with $\mathcal{F}=\mathcal{F}^{u}=\mathcal{F}^{s}$. If $A \notin \operatorname{fin}(S)$, $C \notin \operatorname{fin}(S)^{c c}, A \cap C=\emptyset$ and $A \notin \operatorname{cclass}_{1}(C, \mathcal{F})$, then $B \subseteq A$ exists with $B \in \operatorname{ccore}_{1}(C, \mathcal{F})$.

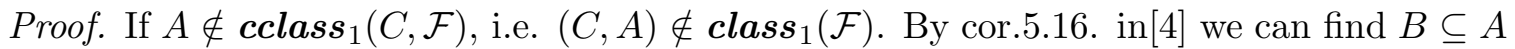
such that for all infinite $B^{\prime} \subseteq B\left(C, B^{\prime}\right) \notin \operatorname{class}_{2}(\mathcal{F})$, i.e. $B \in \operatorname{ccore}_{1}(C, \mathcal{F})$.

Using this lemma in connection with Theorem 4.4. we get

Lemma 4.6. Let $\mathcal{F}$ be denumerable and nontrivial with $\mathcal{F}=\mathcal{F}^{u}=\mathcal{F}^{s}$ and $(C, \boldsymbol{A})$ a conditional classification problem where $C$ and $C^{c}$ are infinite. If $\boldsymbol{A}=\left(A_{1}, \ldots, A_{k}\right)$ with $A_{i} \notin \operatorname{cclass}_{1}(C, \mathcal{F})$ for $1 \leq i \leq k$ then $a \boldsymbol{B} \leq \boldsymbol{A}$ exists with $|\boldsymbol{B}|=k$ and $\boldsymbol{B} \in \operatorname{ccore}_{k}(C, \mathcal{F})$.

Proof. By Lemma 4.5. we find for each $1 \leq i \leq k B_{i} \in$ ccore $_{1}(C, \mathcal{F})$ and $B_{i} \subseteq A_{i}$. Let $\mathbf{B}=\left(B_{1}, \ldots, B_{k}\right)$. Then $\mathbf{B} \leq \mathbf{A}$ and $|\mathbf{B}|=k$. By Theorem 4.4. $\mathbf{B} \in$ ccore $_{k}(C, \mathcal{F})$.

\section{Conditional Cores and Hard Cores}

For WP-recursive language families we can prove a much stronger result. This depends on the relation between $A \in$ ccore $_{1}(C, \mathcal{F})$ and proper hard cores introduced by N. Lynch [6] for complexity classes and in a very general form by R. Book- D.-Z. Du [3].

Definition 5.1. $B$ is a $\mathcal{F}$-hardcore of $A$ if and only if $B$ is infinite and for all $C \in \mathcal{F}(A)$ : $B \cap C \in \boldsymbol{f i n}(S)$. If additionally $B \subseteq A$ then $B$ is a proper $\mathcal{F}$-hardcore of $A$. (Remind $\mathcal{F}(A)=\{Q \subseteq A \mid Q \in \mathcal{F}\}$ for $\mathcal{F}$ and $A$.)

Note, that for $A^{\prime} \subseteq A$ with $A^{\prime}$ infinite every $\mathcal{F}$-hardcore of $A$ is a $\mathcal{F}$-hardcore of $A^{\prime}$. Rephrasing Lemma 7.2. of [4] we get the following

Lemma 5.2. If $\mathcal{F}$ is nontrivial with $\mathcal{F}=\mathcal{F}^{c o}$ and $(C, A)$ a conditional classification problem then $A$ is a proper $\mathcal{F}$-hardcore of $C^{c}$ if and only if $A \in$ ccore $_{1}(C, \mathcal{F})$.

Now we can use a construction for proper hard cores from [3] in a modified form.

Theorem 5.3. If $\mathcal{L}$ is a nontrivial and $W P$-recursive language family with $\mathcal{L}=\mathcal{L}^{b}$ and $(C, A)$ a conditional classification problem with $A \notin \operatorname{cclass}_{1}(C, \mathcal{L})$ and $C, A$ are recursive then a recursive $B \subseteq A$ exists with $B \in$ ccore $_{1}(C, \mathcal{L})$. 
Proof. Consider an enumeration e of $\mathcal{L}$ such that $\boldsymbol{w o r d}_{\mathbf{e}} \in \boldsymbol{r e c}_{2}$. Furthermore, let $\delta_{C}, \delta_{A} \in$ $\boldsymbol{r e c}_{1}$. Now define for all $n \geq 0 B(n), \operatorname{cancel}(n)$ and $\operatorname{card}(n)$ by the following algorithm:

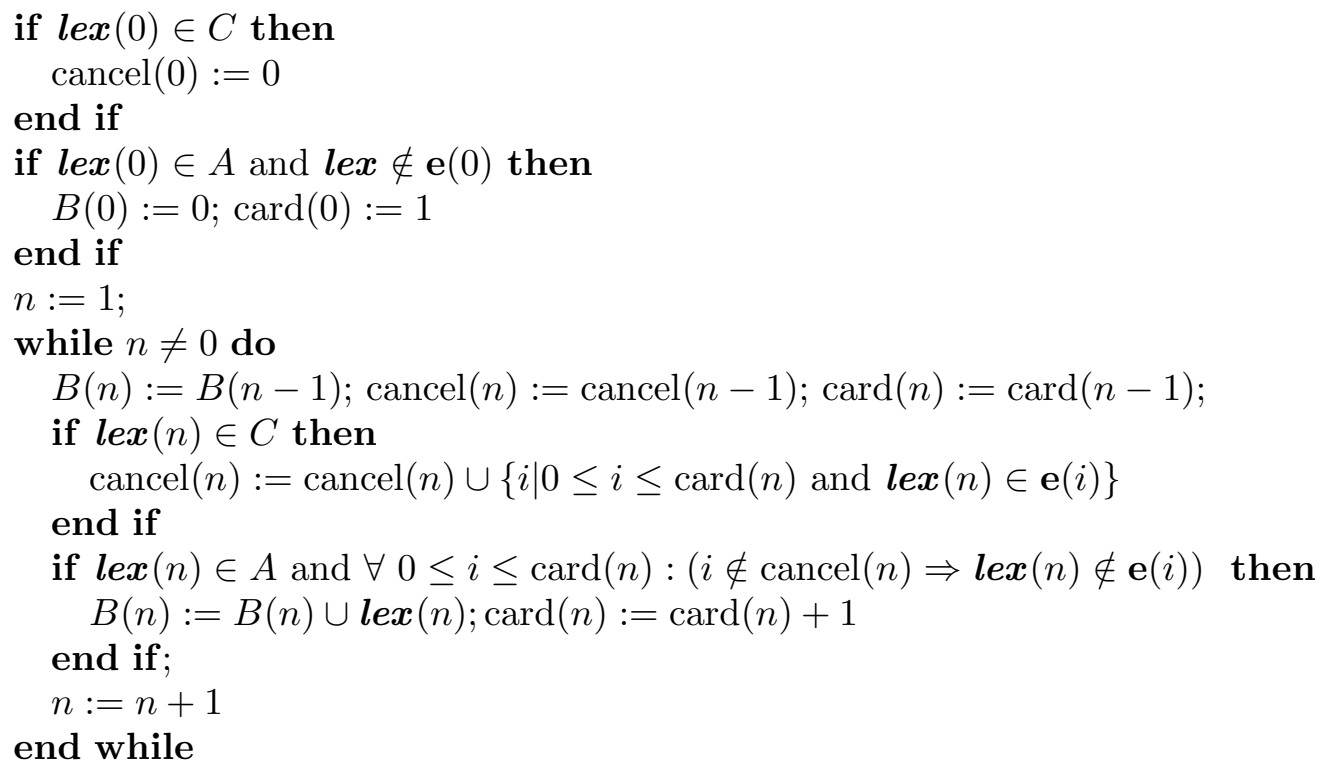

(For $A=C^{\mathbf{c}}$ we get the construction of [3]).

Now, let $B=\bigcup_{i=0}^{\infty} B(n)$ and cancel $=\bigcup_{i=0}^{\infty} \operatorname{cancel}(i)$. Assume for the moment that $B$ is infinite. $B$ is recursive and $B \subseteq A$, since all basic functions are recursive, $\operatorname{cancel}(n)$ is finite for all $n$ and the elements of $B$ are added in increasing order with respect to lex . Moreover, $\lim _{n \rightarrow \infty} \operatorname{card}(n)=\infty$. Hence $\{k \mid \mathbf{e}(k) \cap C \neq \emptyset\}=$ cancel and we get $\mathbf{e}(i) \subseteq C^{\mathbf{c}}$ and by construction $\mathbf{e}(i) \cap B \in \boldsymbol{f i n}\left(X^{*}\right)$ for $i \notin$ cancel (cf. [3]). In conclusion, $B$ is a proper $\mathcal{L}$-hardcore of $C^{\mathbf{c}}$ and by Lemma 4.9. $B \in$ ccore $_{1}(C, \mathcal{L})$. It remains to show the

Assertion: $B \notin \operatorname{fin}\left(X^{*}\right)$.

Suppose to the contrary, that $B$ is finite. Then $M$ exists with $\operatorname{card}(n)=M$ for almost all $n$. Moreover, for every $i \in[M+1]_{0}$ with $\mathbf{e}(i) \cap C \neq \emptyset$ there must exist $K(i)$ with $i \in \operatorname{cancel}(K(i))$. Let $K=\max \left\{K(i) \mid i \in[M+1]_{0}\right.$ with $\left.\mathbf{e}(i) \cap C \neq \emptyset\right\}$. Then we know that for all $i \in[M+1]_{0}$ with $i \notin \operatorname{cancel}(K(i)): \mathbf{e}(i) \subseteq C^{\mathbf{c}}$. Choose $N \geq K$ sufficiently large such that additionally $\operatorname{card}(n)=M$ for every $n \geq N$. Consider $\boldsymbol{l e x}(n) \in A$ with $n \geq N$. Since $\boldsymbol{l e x}(n) \notin B, i \in[M+1]_{0}$ exists with $\boldsymbol{l e x}(n) \in \mathbf{e}(i)$. This shows $A \subseteq\{\boldsymbol{l e x}(k) \mid k<N$ and lex $(k) \in A\} \cup \bigcup_{i=0, i \notin \text { cancel }}^{M} \mathbf{e}(i)=Q \subseteq C^{\mathbf{c}}$ and therefore $C \subseteq Q^{\mathbf{c}}$. Since $\mathcal{L}$ is nontrivial and $\mathcal{L}=\mathcal{L}^{\mathbf{u}}$, we know $Q \in \mathcal{L}$. Moreover, $\mathcal{L}=\mathcal{L}^{\text {co }} \operatorname{implies}^{\mathbf{c}} \in \mathcal{L}$, hence $A \notin \operatorname{cclass}_{1}(C, \mathcal{L})$ - a contradiction.

Now we can derive a stronger result than Lemma 4.6.:

Theorem 5.4. Let $\mathcal{L}$ be a nontrivial and $W P$-recursive language family with $\mathcal{L}=\mathcal{L}^{b}$ and $(C, \boldsymbol{A})$ a conditional $k$-classification problem. If $C$ is recursive and $\boldsymbol{A}=\left(A_{1}, \ldots, A_{k}\right)$ such that $A_{i} \in$ cclass $_{1}(C, \mathcal{L})$ and $A_{i}$ is recursive for $1 \leq i \leq k$ then $\boldsymbol{B}=\left(B_{1}, \ldots, B_{k}\right)$ exists with $\boldsymbol{B} \leq \boldsymbol{A}, \boldsymbol{B} \in$ ccore $_{k}(C, \mathcal{L})$ and $B_{i}$ is recursive for $1 \leq i \leq k$.

Proof. By Theorem 5.3. we find for each $1 \leq i \leq k B_{i} \in \operatorname{cclass}_{1}(C, \mathcal{L})$ with $B_{i} \subseteq A_{i}$ and $B_{i}$ is recursive. Let $\mathbf{B}=\left(B_{1}, \ldots, B_{k}\right)$. Then $\mathbf{B} \leq \mathbf{A}$ and by Theorem 4.4. $\mathbf{B} \in \operatorname{ccore}_{k}(C, \mathcal{L})$. 
Remark 5.5. The $B_{i}$ 's constructed in Theorem 5.4. are all infinite. By the Dekker-Myhill theorem ( $\S 12.3$ Theorem VI in [7]), we can find in every $B_{i}$ a $\mathcal{L}$-cohesive $B_{i}^{\prime}$, but we cannot show, that $B_{i}^{\prime}$ is recursive under the conditions of Theorem 5.4. The best result to our knowledge is the result of Friedberg ( $\$ 12.4$ Theorem XI in [7]). The construction (due to Yates) in the proof given in [7] can be easily modified in such a way, that to any infinite, recursive $A$ a $\mathcal{L}_{\text {r.e. }}(X)$-cohesive subset $B$ with $B^{\mathbf{c}} \in \mathcal{L}_{\text {r.e. }}(X)$ can be found. Since any WP-recursive language family $\mathcal{L}$ is a subfamily of $\mathcal{L}_{\text {r.e. }}(X)$ this $B$ is $\mathcal{L}$-cohesive, too.

\section{CONCLUDing REMARKS}

This paper continues our research about unsolvability cores in promise problems ([4]) generalizing the results to classification problems. Our approach is very general, though the applications in this paper deal mainly with language families and complexity classes. The main open problem in our approach is to construct cohesive sets with "nice" properties.

\section{ACKNOWLEDGment}

We acknowledge seminal discussions with Martin Ziegler.

\section{REFERENCES}

[1 ] K. Ambos-Spies, U. Brandt, M. Ziegler: "Real Benefit of Promises and Advice", pp. 1-11, CiE 2013

[2 ] J.L.Balcazar, J.Diaz, J.Gabarro: "Structural Complexity I" EATCS Monographs on Theoretical Computer Sciences, Springer Verlag (1988)

[3 ] R.V.Book, Ding-Zhu Du: "The Existence and Density of Generalized Complexity Cores", pp.718-730 in JACM vol.34:3 (July 1987).

[4 ] U.Brandt, H.K.-G.Walter: "Cohesiveness in Promise Problems", RAIRO - Theoretical Informatics and Applications vol. 47:4 (November 2013).

[5 ] S.Even, A.L.Selman and Y.Yacobi: "The Complexity of Promise Problems with Applications to Public-Key Cryptography", pp.159-173 in Information and Control vol.61 (1984).

[6 ] N.Lynch: "On Reducibility to Complex or Sparse sets" JACM, vol.3(July 1975), pp.341-345

[7 ] Hartley Rogers Jun.: "Theory of Recursive Functions and Effective Computability", MacGraw-Hill Book Company (1967). 